\title{
A Method for Aggregating Linguistic Preference Relations Based on IOWA
}

\author{
Li Qin ${ }^{1,2}$ Zheng Pei ${ }^{1}$ Nini Duan ${ }^{1}$ \\ ${ }^{1}$ School of Mathematics \& Computer Science, Xihua University, Chengdu 610039, China \\ ${ }^{2}$ Department of Computer, Dezhou University, Dezhou 253023, China
}

\begin{abstract}
$I O W A$ operator, which is proposed by Yager, et al, has been used in many fields. In the paper, we deal with group linguistic assessments decisionmaking problems based on IOWA. Firstly, in the homogeneous, the higher the consistence degree of experts is, the higher the weights of linguistic assessments is, thus we explain the order inducing value of IOW A operator as numbers of the experts who assign the same linguistic assessment; In the heterogeneous, the bigger the importance degree of exports is, the higher the weights of linguistic assessments is, thus we explain the order inducing value of IOW A operator as sum of importance degrees of all experts who assign the same linguistic assessment. Secondly, we use the order inducing values to compute the weights of IOW A operator. Thirdly, we use IOW $A$ operator to aggregate group linguistic preference relations, and utilize linguistic length to dispose 'ties' among linguistic assessments. Finally, we introduce a process of aggregation and give an example.
\end{abstract}

Keywords: IOW A operator, IULOWA operator, $I-I O W A$ operator, Ties, Linguistic preference relations

\section{Introduction}

Based on the Ordered Weighted Averaging ( $O W A$ ) operator, Yager, et al propose the Induced Ordered Weighted Averaging (IOWA) Operators, which extends $O W A$ operator [1]-[5]. These operators take as their argument pairs, called $O W A$ pairs, in which one component called the order inducing variable is used to induce an ordering over another components which are then aggregated. The difference between $O W A$ and IOW $A$ operators is the ordering of aggregated values. The former's ordering is based on the values which will be aggregated. The latter's ordering is decided by the order inducing values [6]. Because of including the order inducing variable, IOW A operator can be used in more complex environments which include linguistic and numeric mixed variables [1]. Several authors have provided some interesting results on IOWA operator, such as, in [7], the author provides an induced uncertain linguistic $O W A(I U L O W A)$ operators in which the aggregated values are uncertain linguistic variables; In [6], the author presents some operators including the Importance $I O W A$ $(I-I O W A)$ operator, which applies the ordering of the argument values based upon the importance of the information sources; the Consistency IOW A $(C-I O W A)$ operator, which applies the ordering of the argument values based upon the consistency of the information sources; and the Preference IOWA $(P-I O W A)$ operator, which applies the ordering of the argument values based upon the relative preference values associated to each one of them.

This paper's structure is arranged as follows. In section 2, we give some basic knowledge about $I O W A$ operators. In section 3 , we develop two new method of IOW A operators to aggregate linguistic preference relations and analyze some properties of the aggregated linguistic preference relation. In section 4 , we present a new way to deal with 'ties'. In section 5, we list steps of aggregation and give an whole example. The conclusions is in section 6 .

\section{Preliminaries}

In this section, we introduce basic knowledge about $I O W A$ operator and extended IOWA operators. We refer to [1]-[6] for more detail. Formally, IOW A operator can be defined as follows.

Definition 1 [6] An IOW A operator of dimension $n$ is a function IOW $A_{W}:(R \times R)^{n} \rightarrow R$, to which a set of weights or weighting vector is associated, $W=\left(w_{1}, \ldots, w_{n}\right)$, such that $w_{i} \in[0,1]$ and $\sum_{i=1}^{n} w_{i}=1$, and it is defined to aggregate the set of second arguments of a list of 2-tuples $\left\{\left\langle u_{1}, p_{1}\right\rangle, \ldots,\left\langle u_{n}, p_{n}\right\rangle\right\}$ according to the following ex- 
pression:

$$
\operatorname{IOW} A_{W}\left(\left\langle u_{1}, p_{1}\right\rangle, \ldots,\left\langle u_{n}, p_{n}\right\rangle\right)=\sum_{i=1}^{n} w_{i} \cdot p_{\sigma(i)},
$$

being $\sigma:\{1, \ldots, n\} \rightarrow\{1, \ldots, n\}$ a permutation such that $u_{\sigma(i)} \geqslant u_{\sigma(i+1)}, \forall i=1, \ldots, n-1$, i.e., $\left\langle u_{\sigma(i)}, p_{\sigma(i)}\right\rangle$ is the 2-tuple with $u_{\sigma(i)}$ the $i$-th highest value in the set $\left\{u_{1}, \ldots, u_{n}\right\}$.

Example 1 Assume there is a set of $O W A$ pairs $\{\langle 0.5,7\rangle,\langle 0.3,9\rangle,\langle 1,5\rangle,\langle 1.2,10\rangle\}$ and a weighting vector $W^{T}=(0.2,0.3,0.4,0.1)$. Firstly we get an ordering of the four $O W A$ pairs according to the first components of $O W A$ pairs, i.e., $1.2>$ $1>0.5>0.3$, the ordering of $O W A$ pairs is $\langle 1.2,10\rangle,\langle 1,5\rangle,\langle 0.5,7\rangle,\langle 0.3,9\rangle$. Then we aggregate the second components and get

$$
\begin{aligned}
& \operatorname{IOW} A_{W}(\langle 0.5,7\rangle,\langle 0.3,9\rangle,\langle 1,5\rangle,\langle 1.2,10\rangle) \\
= & 0.2 \times 10+0.3 \times 5+0.4 \times 7+0.1 \times 9=7.2 .
\end{aligned}
$$

Linguistic labels are the appropriate tools to describe vague concepts in natural language. Assume there is a set of linguistic labels $S=\left\{s_{i} \mid i=\right.$ $1, \ldots,|S|\}$, where $s_{i}$ present a vague concept and if $i<j$, then $s_{i}<s_{j}$. $|S|$ is cardinality of $S$, if $|S|$ is odd number, linguistic labels are placed symmetrically around the middle label $s_{\frac{|S|+1}{2}}$.

Example 2 [7] Let $S=\left\{s_{1}=\right.$ extremely poor, $s_{2}=$ very poor, $s_{3}=$ poor, $s_{4}=$ slightly poor, $s_{5}=$ fair, $s_{6}=$ slightly good, $s_{7}=$ good, $s_{8}=$ very good, $s_{9}=$ extremely good $\} . s_{5}$ is the middle label, the rest are placed symmetrically around $s_{5}$ and there is $s_{1}<s_{2}<\cdots<s_{9}$.

Induced uncertain linguistic $O W A(I U L O W A)$ in [7] is used to aggregate some uncertain linguistic variables.

Definition 2 [7] IULOWA operator is defined as follows:

$$
\begin{aligned}
& \operatorname{IULOW} A_{W}\left(\left\langle u_{1}, s_{1}\right\rangle, \cdots,\left\langle u_{n}, s_{n}\right\rangle\right) \\
= & w_{1} \odot s_{\beta_{1}} \oplus w_{2} \odot s_{\beta_{2}} \oplus \cdots \oplus w_{n} \odot s_{\beta_{n}}
\end{aligned}
$$$$
\text { where } s_{i} \oplus s_{j}=s_{i+j}, \lambda \odot s_{i}=s_{\lambda i} . W^{T}=\left(w_{1}, \ldots\right. \text {, }
$$
$\left.w_{n}\right)$ is a weighting vector, such that $w_{i} \in[0,1]$ and $\sum_{i=1}^{n} w_{i}=1 . s_{\beta_{j}}$ is the $s_{i}$ of the IULOWA pair $\left\langle u_{i}, s_{i}\right\rangle$ having the $j$ th largest $u_{i}$.

During aggregating experts' ideas, there will be some linguistic labels which don't belong to $S$. To preserve all the given information, we extend the discrete term set $S$ to a continuous term set $\bar{S}=\left\{s_{\alpha} \mid \alpha \in[1, t]\right\}$. If $s_{\alpha} \in S$, we call $s_{\alpha}$ an original linguistic term, otherwise, we call $s_{\alpha}$ a virtual linguistic term [8].

Example 3 Assume being a set of $O W A$ pairs $\left\{\left\langle 0.5, s_{7}\right\rangle,\left\langle 0.3, s_{8}\right\rangle,\left\langle 1, s_{5}\right\rangle,\left\langle 1.2, s_{1}\right\rangle\right\}$ and a weighting vector $W^{T}=(0.2,0.3,0.4,0.1)$. Then according to IULOWA operator, the result is:

$$
\begin{aligned}
& I U L O W A_{W}\left(\left\langle 0.5, s_{7}\right\rangle,\left\langle 0.3, s_{8}\right\rangle,\left\langle 1, s_{5}\right\rangle,\left\langle 1.2, s_{1}\right\rangle\right) \\
= & 0.2 \odot s_{1} \oplus 0.3 \odot s_{5} \oplus 0.4 \odot s_{7} \oplus 0.1 \odot s_{8}=s_{5.3}
\end{aligned}
$$

The Importance IOW A $(I-I O W A)$ operator in $[6]$ is used to aggregate fuzzy preference relations.

Definition $3 I-I O W A$ operator is defined as follows:

$$
\begin{aligned}
& I-I O W A_{W}\left(\left\langle u_{1}, P^{1}\right\rangle, \cdots,\left\langle u_{n}, P^{n}\right\rangle\right) \\
= & w_{1} \odot P^{\beta_{1}} \oplus w_{2} \odot P^{\beta_{2}} \oplus \cdots \oplus w_{n} \odot P^{\beta_{n}},
\end{aligned}
$$

where $P^{k}=\left(p_{i j}^{k}\right), p_{i j}^{k} \in[0,1]$, which are additive reciprocal, i.e., $p_{i j}^{k}+p_{j i}^{k}=1 . P^{\beta_{x}} \oplus P^{\beta_{y}}=\left(p_{i j}^{\beta_{x}}+p_{i j}^{\beta_{y}}\right)$ and $\lambda \odot P^{\beta_{x}}=\left(\lambda \times p_{i j}^{\beta_{x}}\right) . \quad P^{\beta_{j}}$ is $P^{i}$ of the $I-I O W A$ pair $\left\langle u_{i}, P^{i}\right\rangle$ having the $j-$ th largest $u_{i}$ which is the importance degree of the export $e_{i}$. $W^{T}=\left(w_{1}, \ldots, w_{n}\right)$ is a weighting vector, such that $w_{i} \in[0,1]$ and $\sum_{i=1}^{n} w_{i}=1 . s_{\beta_{j}}$ is $s_{i}$ of the IULOWA pair $\left\langle u_{i}, s_{i}\right\rangle$ having the $j$ th largest $u_{i}$.

In $I-I O W A$ operator, the importance degree of the information sources or experts are regarded as the order inducing variable. While it is used to compute the weighting vector with a fuzzy language quantifier $Q$. According to the idea that the bigger the importance degree of experts is, the higher the weights of linguistic assessments is, the components of the weighting vector have to be decreasing [6]. i.e., $w_{1}>w_{2}>\cdots>w_{n}$. The following formula is used to compute the weighting vector [9].

$$
\begin{aligned}
w_{k} & =Q\left(\frac{S(k)}{S(n)}\right)-Q\left(\frac{S(k-1)}{S(n)}\right), \\
Q(r) & =r^{a} .
\end{aligned}
$$

where $a \in[0,1], S(k)=\sum_{l=1}^{k} u_{\sigma(l)}$ and $\sigma$ is the permutation such that $u_{\sigma(k)}$ in $\left(u_{\sigma(k)}, p_{i j}^{\sigma(k)}\right)$ is the $k$-th largest value in $\left\{u_{i} \mid u_{i} \in[0,1], i=1, \ldots, n\right\}$.

Example 4 . Suppose three alternatives $\left\{x_{1}, x_{2}\right.$, $\left.x_{3}\right\}$ and three experts $\left\{e_{1}, e_{2}, e_{3}\right\}$, whose importance degree are $\{2.12,1.01,1.37\}$ and whose fuzzy preference relations are: 


$$
\begin{aligned}
P^{1} & =\left(\begin{array}{ccc}
- & 0.75 & 0.87 \\
0.25 & - & 0.66 \\
0.13 & 0.34 & -
\end{array}\right) \\
P^{2} & =\left(\begin{array}{ccc}
- & 0.66 & 0.94 \\
0.34 & - & 0.87 \\
0.06 & 0.13 & -
\end{array}\right) \\
P^{3} & =\left(\begin{array}{ccc}
- & 0.66 & 0.75 \\
0.34 & - & 0.66 \\
0.25 & 0.34 & -
\end{array}\right)
\end{aligned}
$$

then based on above definition 3 and formula 1, having

$$
\begin{aligned}
& I-I O W A_{W}\left(\left\langle 2.12, P^{1}\right\rangle,\left\langle 1.01, P^{2}\right\rangle,\left\langle 1.37, P^{3}\right\rangle\right) \\
& =0.69 \odot P^{1} \oplus 0.19 \odot P^{3} \oplus 0.12 \odot P^{2} \\
& =\left(\begin{array}{ccc}
- & 0.72 & 0.86 \\
0.28 & - & 0.69 \\
0.14 & 0.31 & -
\end{array}\right) .
\end{aligned}
$$

\section{New application of IOWA}

We present a new application associated IULOW A operator with $I-I O W A$ operator, i.e., we use $I O W A$ operator to aggregate linguistic preference relation, and the order inducing values of $I O W A$ operator are explained as the number of experts who argument the same linguistic assessment or the sum of the importance degree of them.

Now, we analyze the characteristics of linguistic preference relation which is given by each expert. Assume a set of alternatives $X=\left\{x_{i} \mid i=1, \cdots, n\right\}$, a set of experts $E=\left\{e_{k} \mid k=1, \cdots, m\right\}$, a set of labels $S=\left\{s_{l} \mid l=1, \cdots, t\right\}$ which are collective of all experts' linguistic preference degree, a set of preference relations $P=\left\{P^{k} \mid k=1, \cdots, m\right\} . p_{i j}^{k} \in P^{k}$ denotes the preference degree of alternative $x_{i}$ over $x_{j}$, it has the following characteristics[12]:

1. $\forall k \in\{1, \cdots, m\}$ and $i \neq j, p_{i j}^{k} \in S$;

2. If $\forall k \in\{1, \cdots, m\}, p_{i j}^{k}=s_{l}$, then $p_{j i}^{k}=$ $N e g\left(s_{l}\right)=s_{t+1-l}$, where $i, j=1, \ldots, n, l=1, \ldots, t$, and

$$
P=\left(\begin{array}{cccc}
- & s_{1} & s_{3} & s_{7} \\
s_{7} & - & s_{2} & s_{4} \\
s_{5} & s_{6} & - & s_{6} \\
s_{1} & s_{4} & s_{2} & -
\end{array}\right)
$$

where $t=7, n=4$, i.e., there are seven labels in the set of $S$ and four alternatives in the set of $X$.

Group decision making $(G D M)$ problems can be roughly classified into two types: the homogeneous and the heterogeneous [10]-[11]. In the first type, the experts' importance degree are equal; In the second type, the experts' importance degree are unequal. $\forall x_{i}, x_{j} \in X, S\left(x_{i}, x_{j}\right)=$ $\left\{s_{l_{1}}, s_{l_{2}}, \cdots, s_{l_{m^{\prime}}}\right\}\left(m^{\prime} \leq m\right)$ represents all experts' linguistic preference degrees for the alternative $x_{i}$ over $x_{j}$. For $s_{l_{m^{\prime \prime}}} \in S\left(x_{i}, x_{j}\right)$, let

$$
v\left(x_{i}, x_{j}, s_{l_{m^{\prime \prime}}}\right)=\sum_{k=1}^{m} \delta\left(p_{i j}^{k}, s_{l_{m^{\prime \prime}}}\right)
$$

a) In homogeneous case, where $\delta\left(p_{i j}^{k}, s_{l_{m^{\prime \prime}}}\right)=1$ if $p_{i j}^{k}=s_{l_{m^{\prime \prime}}}$; Otherwise, $\delta\left(p_{i j}^{k}, s_{l_{m^{\prime \prime}}}\right)=0$.

b) In heterogeneous case, where $\delta\left(p^{k}, s_{l_{m^{\prime \prime}}}\right)=$ $I D_{k}$ if $p_{i j}^{k}=s_{l_{m^{\prime \prime}}}$; Otherwise, $\delta\left(p^{k}, s_{l_{m^{\prime \prime}}}\right)=0, I D_{k}$ is importance degree of $e_{k}$.

In our paper, we use $v_{i j k}$ to denote $v\left(x_{i}, x_{j}, s_{k}\right)$. Next, we introduce the two cases respectively.

(1) The homogeneous case. The exports' importance degrees is equal, so we don't take into account them during aggregation process. $v_{i j k}$ stands for the number of experts who choose the label $s_{k}$ as the preference degree of the alternative $x_{i}$ over $x_{j}$ and $\left\langle v_{i j k}, s_{k}\right\rangle$ is $O W A$ pair. In general, the bigger experts' consensus, the higher the label's weighting vale. So we use (1) and IOWA operator to aggregate the set of $\left\{\left\langle v_{i j k}, s_{k}\right\rangle \mid v_{i j k}>0, k=1, \ldots, t\right\}$, and obtain a collective preference degree of the alternative $x_{i}$ over $x_{j}$. We can get a collective linguistic preference relation $\bar{P}$. i.e,

$$
\begin{gathered}
\bar{p}_{i j}=\operatorname{IOW} A_{W}\left(\left\langle v_{i j 1}, s_{1}\right\rangle, \ldots,\left\langle v_{i j t}, s_{t}\right\rangle\right) \\
\bar{P}=\left(\bar{p}_{i j}\right)
\end{gathered}
$$

Property 1 If the inducing vale $v_{i j k}=0$, then the OW A pair $\left\langle v_{i j k}, s_{k}\right\rangle$ don't join in the calculation of aggregation.

Proof According to (1), if the inducing vale is zero, then the weighting value associated with it is also zero. So we only aggregate the $O W A$ pairs which satisfy with $v_{i j k}>0$.

Property $2 v_{i j k}=v_{j i k^{\prime}}$, in which $k^{\prime}=t+1-k$.

Proof If an expert $e_{h}$ choose $s_{k}$ as the preference degree of $x_{i}$ over $x_{j}$, then according to the characteristics of preference relation, he must choose $s_{k^{\prime}}, k^{\prime}=t+1-k$ as the preference degree of $x_{j}$ over $x_{i}$; vice versa.

Property 3 If $\bar{p}_{i j}=s_{k}$, then $\bar{p}_{j i}=\operatorname{Neg}\left(s_{i}\right)=$ $s_{t+1-k}$; where $i, j=1, \ldots, n ; k=[1, t]$. 
Proof Let $v_{i j 1}>\cdots>v_{i j t}$, then

$$
\begin{aligned}
\bar{p}_{i j} & =I O W A_{W}\left(\left\langle v_{i j 1}, s_{1}\right\rangle,\left\langle v_{i j 2}, s_{2}\right\rangle, \ldots,\left\langle v_{i j t}, s_{t}\right\rangle\right) \\
& =w_{1} \odot s_{1} \oplus w_{2} \odot s_{2} \oplus \cdots \oplus w_{t} \odot s_{t} \\
& =s_{1 \cdot w_{1}} \oplus s_{2 \cdot w_{2}} \cdots \oplus s_{t \cdot w_{t}} \\
& =s_{w_{1} \cdot(t+1-t)+w_{2} \cdot(t+1-(t-1))+\cdots+w_{t} \cdot(t+1-1)} \\
& =s_{\left(w_{1}+w_{2}+\cdots+w_{t}\right) \cdot(t+1)-\left(w_{1} \cdot t+w_{2} \cdot(t-1)+\cdots+w_{t} \cdot 1\right)} \\
& =s_{(t+1)-\left(w_{1} \cdot t+w_{2} \cdot(t-1)+\cdots+w_{t} \cdot 1\right)}
\end{aligned}
$$

Because $\bar{p}_{i j}=s_{k}$, therefore, $t+1-\left(w_{1} \cdot t+w_{2} \cdot(t-\right.$ $\left.1)+\cdots+w_{t} \cdot 1\right)=k \Leftrightarrow w_{1} \cdot t+w_{2} \cdot(t-1)+\cdots+w_{t} \cdot 1=$ $t+1-k$. According to Property 2,

$$
\begin{aligned}
\bar{p}_{j i} & =I O W A_{W}\left(\left\langle v_{j i 1}, s_{1}\right\rangle,\left\langle v_{j i 2}, s_{2}\right\rangle, \ldots,\left\langle v_{j i t}, s_{t}\right\rangle\right) \\
& =I O W A_{W}\left(\left\langle v_{i j t}, s_{1}\right\rangle,\left\langle v_{i j t-1}, s_{2}\right\rangle, \ldots,\left\langle v_{i j 1}, s_{t}\right\rangle\right) \\
& =w_{1} \odot s_{t} \oplus w_{2} \odot s_{t-1} \oplus \cdots \oplus w_{t} \odot s_{1} \\
& =s_{t \cdot w_{1}} \oplus s_{(t-1) \cdot w_{2}} \oplus \cdots \oplus s_{1 \cdot w_{t}} \\
& =s_{t \cdot w_{1}+\cdots+1 \cdot w_{t}}=s_{t+1-k}=\operatorname{Neg}\left(s_{k}\right)
\end{aligned}
$$

Example 5 Assume four exports $E=\left\{e_{1}, e_{2}, e_{3}\right.$, $\left.e_{4}\right\}$, four alternatives $X=\left\{x_{1}, x_{2}, x_{3}, x_{4}\right\}$, seven labels $S=\left\{s_{i} \mid i=1, \ldots, 7\right\}$, and exports give four preference relations $P=\left\{P^{1}, P^{2}, P^{3}, P^{4}\right\}$. If there are one export to choose $s_{1}$ and three exports to choose $s_{4}$ about the preference degree of the alternative $x_{i}$ over $x_{j}$. Then According to Property 1 and 3, having:

$$
\begin{aligned}
\bar{p}_{i j} & =I O W A_{W}\left(\left\langle 1, s_{1}\right\rangle,\left\langle 3, s_{4}\right\rangle\right) \\
& =w_{1} \odot s_{4} \oplus w_{2} \odot s_{1} \\
& =0.93 \odot s_{4} \oplus 0.07 \odot s_{1}=s_{3.79} \\
\bar{p}_{j i} & =s_{4.21}
\end{aligned}
$$

(2) The heterogeneous case. The experts have unequal importance degrees. $v_{i j k}$ stands for the sum of experts' importance degree who choose the label $s_{k}$ as the preference degree of the alternative $x_{i}$ over $x_{j}$. Excepting the meaning of $v_{i j k}$, the rest are similar to the homogeneous case.

Property 4 If the inducing vale $v_{i j k}=0$, then the OWA pair $\left\langle v_{i j k}, s_{k}\right\rangle$ don't join in the calculation of aggregation.

Property $5 v_{i j k}=v_{j i k^{\prime}}, k^{\prime}=t+1-k$.

Property $6 \bar{P}$ is relatively complementary about labels' subscript. i.e. if $\bar{p}_{i j}=s_{k}$, then $\bar{p}_{j i}=$ $\operatorname{Neg}\left(s_{i}\right)=s_{t+1-k} ;$ where $i, j=1, \ldots, n ; k=[1, t]$.

Example 6 Assume four experts $E=\left\{e_{1}, e_{2}, e_{3}\right.$, $\left.e_{4}\right\}$, four alternatives $X=\left\{x_{1}, x_{2}, x_{3}, x_{4}\right\}$, seven labels $S=\left\{s_{i} \mid i=1, \ldots, 7\right\}$, and experts give four preference relations $P=\left\{P^{1}, P^{2}, P^{3}, P^{4}\right\}$, experts' importance degree are $D=\left\{d_{1}=0.5, d_{2}=\right.$ $\left.0.3, d_{3}=0.8, d_{4}=0.1,\right\}$. if experts $e_{1}, e_{2}, e_{4}$ choose $s_{4}$ and expert $e_{3}$ choose $s_{1}$ as the preference degree of $x_{i}$ over $x_{j}$, then according to Property 4 and 6 , having:

$$
\begin{aligned}
\bar{p}_{i j} & =\operatorname{IOW} A_{W}\left(\left\langle v_{i j 1}, s_{1}\right\rangle,\left\langle v_{i j 4}, s_{4}\right\rangle\right) \\
& =\operatorname{IOW} A_{W}\left(\left\langle 0.8, s_{1}\right\rangle,\left\langle 0.9, s_{4}\right\rangle\right) \\
& =w_{1} \odot s_{4} \oplus w_{2} \odot s_{1} \\
& =0.728 \odot s_{4} \oplus 0.272 \odot s_{1}=s_{3.184} . \\
\bar{p}_{j i} & =s_{4.816}
\end{aligned}
$$

\section{A way of dealing with "tie"}

When we use IOWA operator to aggregate words or numbers, we may meet a phenomenon that there are the same inducing values with regard to $O W A$ pairs. We call the phenomenon as "tie". Next, we introduce the following three kind of method.

(1) For ensuring the consistence of the aggregated result, Yager, et al present a method, that is to replace each aggregated component of the tied $O W A$ pairs using their average values [1]. For example, there are four $O W A$ pairs: $\left\langle u_{1}, x_{1}\right\rangle,\left\langle u_{2}, x_{2}\right\rangle,\left\langle u_{3}, x_{3}\right\rangle,\left\langle u_{1}, x_{4}\right\rangle$, where $\left\langle u_{1}, x_{1}\right\rangle$ and $\left\langle u_{1}, x_{4}\right\rangle$ is the tied IOWA pairs. Using the method, firstly we change the four $O W A$ pairs to $\left\langle u_{1}, \frac{x_{1}+x_{4}}{2}\right\rangle,\left\langle u_{2}, x_{2}\right\rangle,\left\langle u_{3}, x_{3}\right\rangle,\left\langle u_{1}, \frac{x_{1}+x_{4}}{2}\right\rangle$, then aggregate them.

(2) Similarly, we develop another method. Firstly, we order $O W A$ pairs on the basis of the order inducing variables. Then we compute the average value of the weighting variables with regard to the tied $O W A$ pairs and replace the them. Assume the four $O W A$ pairs are $\left\langle u_{1}, x_{1}\right\rangle,\left\langle u_{2}, x_{2}\right\rangle,\left\langle u_{3}, x_{3}\right\rangle,\left\langle u_{1}, x_{4}\right\rangle$ and $u_{1}<u_{2}<$ $u_{3}$, the weighting vector is $W=\left(w_{1}, w_{2}, w_{3}, w_{4}\right)$. According to our idea, the weighting vector is changed to $W=\left(w_{1}, w_{2}, \frac{w_{3}+w_{4}}{2}, \frac{w_{3}+w_{4}}{2}\right)$. The above two methods are the same. We can proof:

$$
\begin{aligned}
& \operatorname{IOW} A_{W}\left(\left\langle u_{1}, x_{1}\right\rangle,\left\langle u_{2}, x_{2}\right\rangle,\left\langle u_{3}, x_{3}\right\rangle,\left\langle u_{1}, x_{4}\right\rangle\right) \\
= & w_{1} \cdot x_{3}+w_{2} \cdot x_{2}+w_{3} \cdot \frac{x_{1}+x_{4}}{2}+w_{4} \cdot \frac{x_{1}+x_{4}}{2} \\
= & w_{1} \cdot x_{3}+w_{2} \cdot x_{2}+\left(w_{3}+w_{4}\right) \cdot \frac{x_{1}+x_{4}}{2} \\
= & w_{1} \cdot x_{3}+w_{2} \cdot x_{2}+\frac{\left(w_{3}+w_{4}\right)}{2} \cdot\left(x_{1}+x_{4}\right) \\
= & w_{1} \cdot x_{3}+w_{2} \cdot x_{2}+\frac{\left(w_{3}+w_{4}\right)}{2} \cdot x_{1}+\frac{\left(w_{3}+w_{4}\right)}{2} \cdot x_{4}
\end{aligned}
$$

(3) In the paper, we use IOWA operator to aggregate language labels. Because the special setting, we give another way which is different from 
the former two methods. We think the new way is more suitable for our setting. Next, we explain the new method.

Definition $4 \operatorname{Len}\left(s_{i}, s_{j}\right)=|i-j|$ stand for the length between $s_{i}$ and $s_{j}$. where $s_{i}, s_{j} \in \bar{S}$ and $\bar{S}=$ $\left\{s_{i} \mid i \in[0,1]\right\}$ is a set of language items.

Assume there are the tied $O W A$ pairs $\left\langle u_{i}, s_{i}\right\rangle$, and $\left\langle u_{j}, s_{j}\right\rangle . \quad u_{k}$ is the biggest order inducing value, it's $O W A$ pair is $\left\langle u_{k}, s_{k}\right\rangle$. if $\operatorname{Len}\left(s_{k}, s_{i}\right)>$ $\operatorname{Len}\left(s_{k}, s_{j}\right)$, then $s_{j}$ is in the front of $s_{i}$ in the ordering; Otherwise, $s_{i}$ is in the front of $s_{j}$. When $k(k>2)$ items are tied, we also arrange them ascendingly by their length. It's the consistent with the commonly sense.

When we use the method to deal with the tied pairs, there may be three cases. The one, all the $O W A$ pairs are tied pairs; The second, there still exist the tied pairs after using (3); The third, there is not the biggest order inducing value. When we meet the three cases, we can utilize (1) or (2) to deal with them.

Example 7 Assume there are four experts $E=$ $\left\{e_{1}, e_{2}, e_{3}, e_{4}\right\}$, four alternatives $X=\left\{x_{1}, x_{2}, x_{3}\right.$, $\left.x_{4}\right\}$, seven labels $S=\left\{s_{i} \mid i=1, \ldots, 7\right\}$, four preference relations $P=\left\{P^{1}, P^{2}, P^{3}, P^{4}\right\}$. One expert chooses $s_{1}$, two experts choose $s_{4}$, one expert chooses $s_{6}$ as the preference degree of $x_{i}$ over $x_{j}$ in homogeneous case. we compute the value of $\bar{p}_{i j}$.

Firstly, we find out the objects of aggregation. They are $\left\langle 1, s_{1}\right\rangle,\left\langle 2, s_{4}\right\rangle,\left\langle 1, s_{6}\right\rangle .\left\langle 1, s_{1}\right\rangle$ and $\left\langle 1, s_{6}\right\rangle$ are the tied pairs. Then we use the third way to dispose them. $s_{4}$ is the first label in the ordering. $\operatorname{Len}\left(s_{4}, s_{1}\right)=3, \operatorname{Len}\left(s_{4}, s_{6}\right)=2$, so $s_{6}$ is in the front of $s_{1}$. Using formula 1 and 3, we can obtain

$$
\begin{aligned}
\bar{p}_{i j} & =w_{1} \odot s_{4} \oplus w_{2} \odot s_{6} \oplus w_{3} \odot s_{1} \\
& =s_{0.707 \times 4+0.159 \times 6+0.134 \times 1}=s_{3.916}
\end{aligned}
$$

\section{Example}

Firstly, we give the steps to aggregate language preference relations.

1. Found out $O W A$ pairs with respect to $\bar{p}_{i j}$.

2. Dispose the tied pairs.

3. Compute weighting values according to (1).

4. Compute $\bar{p}_{i j}$ on the basis of (3).

5. Compute the $\bar{P}$.

6. Compute the collective estimation of every alternative according to $P_{i}=\sum_{j=1}^{n} \bar{p}_{i j}$.

7. We obtain the best alternatives $x_{a}$ according to $P_{a}=\max \left\{P_{i} \mid i=1, \ldots, n\right\}$.
A set of labels $S$, a set of alternatives $X$, a set of experts $E$ and a set of preference relations $P$ are as following

$$
\begin{aligned}
& S=\left\{s_{1}, s_{2}, s_{3}, s_{4}, s_{5}, s_{6}, s_{7}\right\} \\
& X=\left\{x_{1}, x_{2}, x_{3}, x_{4}\right\} ; \\
& E=\left\{e_{1}, e_{2}, e_{3}, e_{4}\right\} ; \\
& P=\left\{P^{1}, P^{2}, P^{3}, P^{4}\right\} ; \\
& P^{1}=\left(\begin{array}{llll}
- & s_{1} & s_{6} & s_{2} \\
s_{7} & - & s_{3} & s_{2} \\
s_{2} & s_{5} & - & s_{5} \\
s_{6} & s_{6} & s_{3} & -
\end{array}\right) \\
& P^{2}=\left(\begin{array}{llll}
- & s_{3} & s_{5} & s_{2} \\
s_{5} & - & s_{4} & s_{4} \\
s_{3} & s_{4} & - & s_{5} \\
s_{6} & s_{4} & s_{3} & -
\end{array}\right) \\
& P^{3}=\left(\begin{array}{llll}
s_{7} & s_{5} & s_{2} \\
s_{1} & - & s_{5} & s_{4} \\
s_{3} & s_{3} & - & s_{5} \\
s_{6} & s_{4} & s_{3} & -
\end{array}\right) \\
& P^{4}=\left(\begin{array}{llll}
s_{3} & s_{4} & s_{2} \\
s_{5} & - & s_{6} & s_{2} \\
s_{4} & s_{2} & - & s_{5} \\
s_{6} & s_{6} & s_{3} & -
\end{array}\right)
\end{aligned}
$$

According to the steps of aggregation, we choose the best options under the homogeneous case and assume $a=0.5$ of (1). Aggregation process is following.

1. There are three $O W A$ pairs: $\left\langle 1, s_{1}\right\rangle,\left\langle 2, s_{3}\right\rangle$, $\left\langle 1, s_{7}\right\rangle$ with respect to $\bar{p}_{12}$.

2. After disposing the tied pairs, the $O W A$ pairs' ordering is: $\left\langle 2, s_{3}\right\rangle\left\langle 1, s_{1}\right\rangle\left\langle 1, s_{7}\right\rangle$.

3. The weighting values is $w_{1}=0.707, w_{2}=$ $0.159, w_{3}=0.134$.

4. $\bar{p}_{i j}=3.218$.

5. We get the $\bar{P}$ as following

$$
\bar{P}=\left(\begin{array}{cccc}
- & s_{3.218} & s_{5} & s_{2} \\
s_{4.632} & - & s_{4.5} & s_{3} \\
s_{3} & s_{3.5} & - & s_{5} \\
s_{6} & s_{5} & s_{3} & -
\end{array}\right) .
$$

6. The collective estimation of every alternative is: $x_{1}=10.368, x_{2}=12.132, x_{3}=$ $11.5, x_{4}=14$.

7. The best option is $x_{4}$.

\section{Conclusion}

In the paper, we use $I O W A$ operator to aggregate linguistic preference relation. According to the idea that the bigger the consistence degree of experts in the homogeneous or the importance degree of experts in the heterogeneous, the higher the weighting values, we develop a new method to aggregate language items, while we also introduce a suitable way 
to deal with "tie". And to predigest the calculation, we analyze the properties of $v_{i j}$ and $\bar{P}$. Finally, we give the whole aggregation process. When we compute the aggregation values, we can use the method of $L O W A$ operator that directly compute the labels instead of the method of calculate the labels' subscripts.

\section{Acknowledgement}

This work is supported by the Excellent Young Foundation of Sichuan Province (Grant No.06ZQ026-037), the National Natural Science Foundation of China (60474022), the Special Research Funding to Doctoral Subject of Higher Education Institutes in China (Grant No.20060613007) and a Project Supported by Scientific Research Fund of Sichuan Provincial Education Department(Grant No.2005A121, 2006A084).

\section{References}

[1] R. R. Yager, D. P. Filev, Operations for granular computing: Mixing words and numbers, Prof. the FUZZ-IEEE World Congress on Computational Intelligence, Anchorage, 1998.

[2] R. R. Yager, On induced aggregation operators, in: Prof the EUROFUSE Workshop on Preference Modelling and Applications, Granada, 2000.

[3] R. R. Yager, The induced fuzzy integral aggregation operator. International Journal of Intelligent Systems, 17:1049-1065, 2002.

[4] R. R. Yager, Induced aggregation operators. Fuzzy Sets and Systems, 137:59-69, 2003.

[5] R. R. Yager, D. P. Filev, Induced ordered weighted averaging operators. IEEE Transaction on Systems, Man and Cybernetics, 29:141150, 1999.

[6] F. Chiclana, E. Herrera-Viedma, F. Herrera and S. Alonso, Some induced ordered weighted averaging operators and their use for solving group decision-making problems based on fuzzy preference relations, Eur. J. Oper. res, 182(1):383399, 2007.

[7] Z. S. Xu, Induced uncertain linguistic OWA operators applied to group decision making. Information Fusion, 7:231-238, 2006.

[8] Z. S. Xu, Group decision making based on multiple types of linguistic preference relations . Information Sciences, in press.
[9] R. R. Yager, Quantifier guided aggregation using OWA operators. International Journal of Intelligent Systems, 11:49-73, 1996.

[10] F. Herrera, E. Herrera-Viedma, Aggregation operators for linguistic weighted information. IEEE Transactions on Systems, Man, and Cybernetics Part A: Systems and Humans, 27:646656, 1997.

[11] F. Herrera, E. Herrera-Viedma, J. L. Verdegay, Choice processes for non-homogeneous group decision making in linguistic setting. Fuzzy Sets and Systems, 94:287-308, 1998.

[12] F. Herrera, E. Herrera-Viedma, J. L. Verdegay, Direct approach processes in group decision making using linguistic OWA operators. Fuzzy Sets and Systems, 79:175-190, 1996. 